\title{
Lipids in suspended matter and sediments from the East China Sea Shelf
}

\author{
Woei-Lih Jeng ${ }^{\mathrm{a}, *}$, Chih-An Huh ${ }^{\mathrm{b}}$ \\ ${ }^{a}$ Institute of Oceanography, National Taiwan University, Taipei, Taiwan, ROC \\ ${ }^{\mathrm{b}}$ Institute of Earth Sciences, Academia Sinica, Taipei, Taiwan, ROC \\ Received 28 June 2002; accepted 10 December 2003 \\ (returned to author for revision 9 January 2003)
}

\begin{abstract}
Total suspended matter (TSM) and sediment samples from the East China Sea (ECS) Shelf were analyzed for aliphatic hydrocarbons, alkanols and sterols. TSM samples showed a strong predominance of $n$ - $\mathrm{C}_{17}$, pristane, $n$ - $\mathrm{C}_{18}, n-\mathrm{C}_{19}, n-\mathrm{C}_{19: 1}, n-\mathrm{C}_{21: 6}$ and squalene, being attributed to plankton inputs. Stations with high percentages of these hydrocarbons were generally situated near the Changjiang River mouth. Three stations with highest concentrations of $n$ - $\mathrm{C}_{21: 6}$ were located in the hot spot of chlorophyll reported in the literature (Gong et al., 1996, Continental Shelf Research 16, 1561-1590). Phytol (the most abundant alcohol in TSM) and $\mathrm{C}_{20: 1}$ and $\mathrm{C}_{22: 1} n$-alkenols were dominant in the alcohol fraction, being representative of plankton contributions. The sterol composition of TSM was dominated by

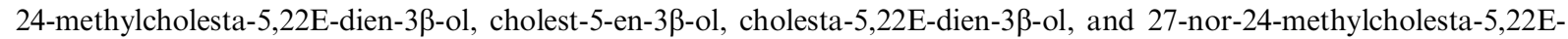

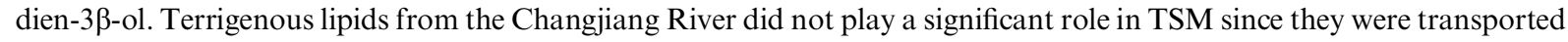
mainly southward. The sedimentary composition of these lipids showed a significant depletion of planktonic compounds in relation to other organic molecules. A comparison of these planktonic hydrocarbons in TSM and in sediments showed that degradation is in the following order: $n-\mathrm{C}_{21: 6}>n-\mathrm{C}_{19: 1}, n-\mathrm{C}_{19}$, and $n-\mathrm{C}_{17}>n-\mathrm{C}_{18}$, pristane, and squalene. A similar comparison for the alcohols showed the following degradation trend: $\mathrm{C}_{20: 1}$ and $\mathrm{C}_{22: 1} n$-alkenols $>$ phytol. Carbon preference indices (CPIs) of suspended matter varied from 1.04 to 1.82 (average 1.41), and those of sediment ranged between 1.82 and 3.74 (average 2.81), reflecting higher contributions of the more refractory higher plant $n$-alkanes in the sediments.
\end{abstract}

(C) 2004 Elsevier Ltd. All rights reserved.

\section{Introduction}

The main circulation patterns of the Changjiang Estuary and adjacent East China Sea (ECS) can be characterized by the northward flow of warm $\left(13{ }^{\circ} \mathrm{C}\right)$ and saline (34 psu) waters of the Taiwan Warm Current (TWC) and a southward flow of the colder $\left(5^{\circ} \mathrm{C}\right)$ and

* Corresponding author. Tel.: + 886-2-23636040 × 301; fax: $+886-2-23626092$.

E-mail address: wljeng@oc.ntu.edu.tw (W.-L. Jeng). less saline (30 psu) waters of the Yellow Sea Coastal Current (YSCC) (Fig. 1). During high river runoff, one part of the Changjiang plume with freshest water extends to the south along the coast, and the other part with low salinity extends offshore toward the northeast on average. However, during low river runoff, the surface plume only spreads toward the south (Beardsley et al., 1985). The Changjiang River annually discharges $\sim 5 \times 10^{8}$ tons of sediment directly into the ECS (Milliman and Meade, 1983). This sediment is confined to the coastal zones of the ECS, being ultimately transported south and southwestward by the Changjiang 
Coastal Water (Milliman et al., 1985). Offshore transport is hindered by tidal currents and by the northward movement of the Taiwan Warm Water (Milliman et al., 1989).

Surface concentrations of suspended particles in the ECS generally range from 1 to $100 \mathrm{mg} / \mathrm{l}$ in winter and 0.5 to $5 \mathrm{mg} / \mathrm{l}$ in summer. In winter near-bottom concentrations tend to be considerably higher than surface values, largely due to resuspension of bottom sediments during intense winter storms (Millman et al., 1989).

A detailed study of summer chlorophyll $a$ distribution indicates that there is a hot spot of chlorophyll $a$ in seawater on the mid-shelf of the ECS (Gong et al., 1996). Previous studies on non-aromatic hydrocarbons in suspended matter from the Changiiang Estuary (Qiu et al., 1991) showed that anthropogenic and/or petrogenic inputs were distributed around the river mouth, being more widespread in winter than in summer. On the other hand, Sicre et al. (1993a) studied $n$-alkanes and polycyclic aromatic hydrocarbons in the suspended particles from the Changiiang Estuary, and observed that terrestrial and anthropogenic inputs prevailed. Bouloubassi et al. (2001) examined hydrocarbons in surface sediments from the Changjiang Estuary and concluded that the overall levels of anthropogenic hydrocarbons were low in comparison to other areas worldwide. Other studies in the Changjiang Estuary and adjacent ECS were devoted to unraveling the terrigenous and marine sterols (Tian et al., 1992; Sicre et al., 1993b, 1994).

Having this previous research in mind, the present study clarifies what are the main sources contributing to the lipid composition of suspended water particles and sediments of the ECS. In addition, the influence of the contributions from the Changjiang River to the ECS will be assessed from the study of alkanes, alcohols and sterols.

\section{Experimental}

\subsection{Sampling}

Eleven stations from the ECS mid-shelf were chosen for sampling surface water $(5 \mathrm{~m})$, and nine stations for surface sediments (Fig. 1). Sampling was performed

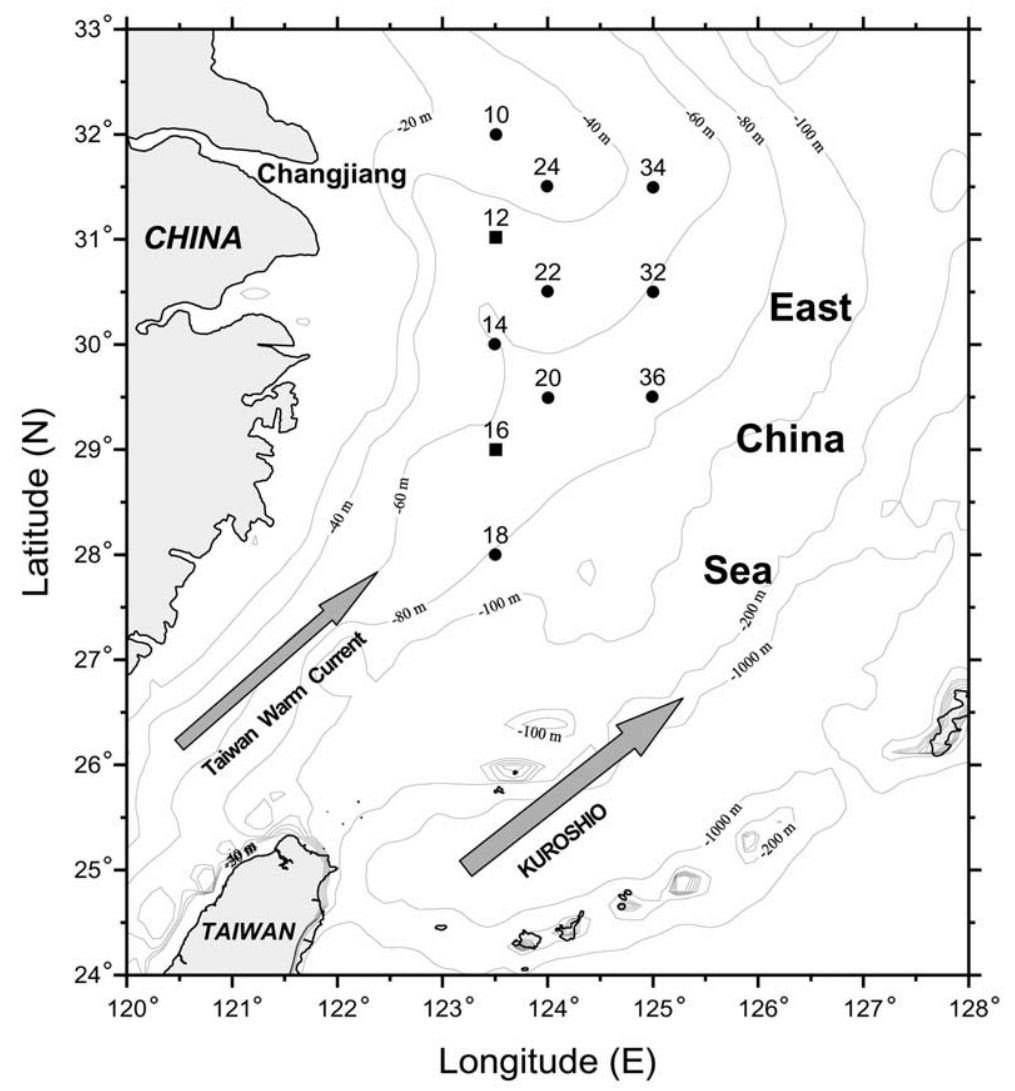

Fig. 1. Sampling sites on the East China Sea Shelf. Solid circles denote collection of both seawater and sediment samples; solid squares denote collection of water samples only. Contours are given in meters. 
between 29 May and 4 June 1999. Surface water (40-50 1) was collected with Niskin bottles on a rosette sampler. Water was filtered in situ through glass fiber filters (Whatman GF/F, $142 \mathrm{~mm}$ diameter) right after sampling. The filters were batch-washed with acetone, methanol, and ethyl acetate several times prior to use. The particulate-laden filters were stored at $-20{ }^{\circ} \mathrm{C}$ until analysis. Sediment samples were collected with a box corer. The top (ca. $3 \mathrm{~cm}$ ) sediment was sliced and kept at $-20{ }^{\circ} \mathrm{C}$ until sample work-up.

\subsection{Sample preparation}

Wet TSM-laden filters were freeze-dried. After adding internal standards $\left(n-\mathrm{C}_{24} \mathrm{D}_{50}\right.$ and 1-heptadecanol), dried TSM samples were extracted with a mixture of dichloromethane and methanol $(1: 1, \mathrm{v} / \mathrm{v})$ for $24 \mathrm{~h}$ in a Soxhlet apparatus. The lipid extract was then saponified by reflux for $3 \mathrm{~h}$ with $0.5 \mathrm{~N} \mathrm{KOH}$ solution in methanol. The non-saponifiable lipids were separated by $n$-hexane extraction four times and concentrated using $\mathrm{N}_{2}$ gas. The aliphatic hydrocarbon fraction and the fraction containing fatty alcohols and sterols were isolated from the neutral lipids by silica gel (5 g, deactivated with $5 \%$ $\left.\mathrm{H}_{2} \mathrm{O}\right)$ column chromatography using $n$-hexane $(20 \mathrm{ml})$ and a mixture of dichloromethane/methanol (4/1, v/v, $40 \mathrm{ml}$ ), respectively. The lipids between the two fractions were eluted with $n$-hexane/dichloromethane $(2 / 3$, $\mathrm{v} / \mathrm{v}, 30 \mathrm{ml}$ ) and discarded. The isolated alcohols and sterols were taken to dryness, redissolved in benzene, and derivatized with N,O-bis(trimethylsilyl)acetamide.

\subsection{Analysis by gas chromatography}

An HP 5890A gas chromatograph equipped with a split/splitless injector and a flame ionization detector (FID) was used for gas chromatographic analysis. An SGE (Australia) OCI-5 cool on-column injector was also fitted in the gas chromatograph. Aliphatic hydrocarbons were separated with an SPB-1 capillary column (30 $\mathrm{m} \times 0.25 \mathrm{~mm}$ i.d.), and alkanols/sterols (as TMS ethers) with an SPB-1 column $(25 \mathrm{~m} \times 0.25 \mathrm{~mm}$ i.d.). Oven temperature programming was $45-90^{\circ} \mathrm{C}$ at $15^{\circ} \mathrm{C} /$ min and $90-270{ }^{\circ} \mathrm{C}$ at $3{ }^{\circ} \mathrm{C} / \mathrm{min}$ for aliphatic hydrocarbons, and $45-90{ }^{\circ} \mathrm{C}$ at $15{ }^{\circ} \mathrm{C} / \mathrm{min}$ and $90-250{ }^{\circ} \mathrm{C}$ at $3{ }^{\circ} \mathrm{C} / \mathrm{min}, 30 \mathrm{~min}$ at $250{ }^{\circ} \mathrm{C}$ for alkanols/sterols. Identification was made with co-injection of authentic standards and gas chromatography-mass spectrometry (GCMS). Compound quantitation was performed using internal standards. Based on replicate analyses, the analytical precision of lipids was calculated to be $2-8 \%$. The GC-MS analyses were performed with an HP 6890 GC (HP-1MS crosslinked methyl siloxane column, $30 \mathrm{~m}$ $\times 0.25 \mathrm{~mm}$ i.d.) interfaced directly to an HP 5973 quadruple mass selective detector (electron impact, electron energy $70 \mathrm{eV}$, scanned from 50 to 550 Daltons).

\section{Results and discussion}

\subsection{Total suspended matter}

\subsubsection{Aliphatic hydrocarbons}

A representative GC trace of aliphatic hydrocarbons from suspended matter is shown in Fig. 2. Seven predominant components were $n-\mathrm{C}_{17}$, pristane, $n-\mathrm{C}_{18}$, $n-\mathrm{C}_{19: 1}, n-\mathrm{C}_{19}, n-\mathrm{C}_{21: 6}$ and squalene; other $n$-alkanes up to $\mathrm{C}_{35}$ were minor. This pattern was similar to the one found at station 1 in DH2 reported by Qiu et al. (1991) although in this latter case squalene was not found. The 7 hydrocarbons are produced chiefly from plankton (Winters et al., 1969; Blumer et al., 1971) and for simplicity are termed planktonically-derived hydrocarbons (PDHCs) in the present study. High relative percentages $(80-94 \%)$ of the seven PDHCs were observed in the hydrocarbon distributions of stations 10, 12, 14, 16, 22, 24,32 and 36, and relatively low percentages (45-61\%) were found at stations 18, 20 and 34 (Table 1). Thus the stations with high percentages of PDHCs were located close to the Changjiang River. In contrast, a very different aliphatic hydrocarbon distribution pattern was obtained in a previous study performed during high flow in September 1988 (Sicre et al., 1993a). In this case, there was no predominance of the 7 PDHCs, and $n$ $\mathrm{C}_{19: 1}, n-\mathrm{C}_{21: 6}$ and squalene were absent in the Changjiang Estuary. Relatively lower percentages (range 6$27 \%$, average $13 \%$, calculated from their data) of PDHCs were obtained in this previous case. Another study reported the occurrence of five types of aliphatic hydrocarbon distributions in the Changjiang Estuary by Qiu et al. (1991). The results of the present study, based on samples located farther off shore, only show two distributions of $n$-alkanes that can be differentiated by the proportion of PDHC's, one with high amounts of these alkanes and alkenes, and the other by near equal concentrations of $n$-alkanes. In any case, the present result suggests a weaker influence of the anthropogenic and riverine inputs than in the previous studies.

In general both photosynthetic algae and bacteria contain $n-\mathrm{C}_{17}$ or pristane as the most abundant hydrocarbon component (Oro et al., 1967; Han et al., 1968; Winters et al., 1969). Non-photosynthetic bacteria may have hydrocarbon distributions dominated by other $n$ alkanes such as $n-\mathrm{C}_{18}$ in $E$. coli, $n-\mathrm{C}_{19}$ in Micrococcus lysodeikticus, and $n-\mathrm{C}_{20}$ in yeast (Han et al., 1968). Seven samples (stations 12, 16, 18, 20, 22, 32 and 34) showed higher abundance of $n-\mathrm{C}_{18}$ or $n-\mathrm{C}_{19}$ over $n-\mathrm{C}_{17}$ (Table 1), which suggests a higher bacterial contribution in these samples. $n-\mathrm{C}_{19: 1}$ accounts for $85-98 \%$ of hydrocarbons in three species of marine blue-green algae (Winters et al., 1969) and occurs in some zooplankton species (Eucalanus sp.) (Saliot, 1981). Higher concentrations of $n-\mathrm{C}_{19: 1}$ than $n-\mathrm{C}_{17}$ were observed in 6 samples (stations 10, 18, 20, 22, 32 and 34) (Table 1), 
and contribution from blue-green algae and copepods is suggested.

The compound cis-heneicosahexa-3,6,9,12,15,18-ene $\left(n-\mathrm{C}_{21: 6}\right)$ is known to predominate in five classes of marine phytoplankton, notably (but not exclusively) diatoms (Blumer et al., 1971; Nichols et al., 1988) and has been generally related to primary productivity (Schultz and Quinn, 1977; Osterroht and Petrick, 1982). The highest concentrations in $n-\mathrm{C}_{21: 6}(56.7,48.5$ and 104 $\mu \mathrm{g} / \mathrm{g}$ at stations 22,24 and 32, respectively) were located within the "hot spot of chlorophyll $a$ ", defined from the observation of chlorophyll $a$ concentrations of $0.2 \mathrm{mg}$ $\mathrm{m}^{-3}$ (Gong et al., 1996). These two concurrent findings suggest the occurrence of phytoplankton bloom every summer at this site.

The abundances of PDHCs were statistically compared to test common sources. Because the data are not normally distributed and include outliers, the Spearman rank coefficient of correlation is the more appropriate statistical test. With this type of correlation, data are measured on a ranking scale rather than the equidistant scale used in the Pearson's product-moment correlation. The Spearman rank coefficients of correlation for the 7 PDHCs are given in Table 2, in which tied ranks were assigned to the less-thans. As seen in Table 2, the 7 PDHCs were not well correlated, suggesting diverse plankton sources. However, $n-\mathrm{C}_{17}, n-\mathrm{C}_{18}$, $n$ - $\mathrm{C}_{19: 1}$ and $n-\mathrm{C}_{19}$ were significantly correlated, possibly indicating that they may derive mainly from nanoplankton and picoplankton. As noted by Wakeham and Lee (1989), small particles collected by in situ filtration in the epipelagic zone are similar in composition to phytoplankton. On the other hand, the correlation of $n$ $\mathrm{C}_{21: 6}$, pristane and squalene suggests a common source,

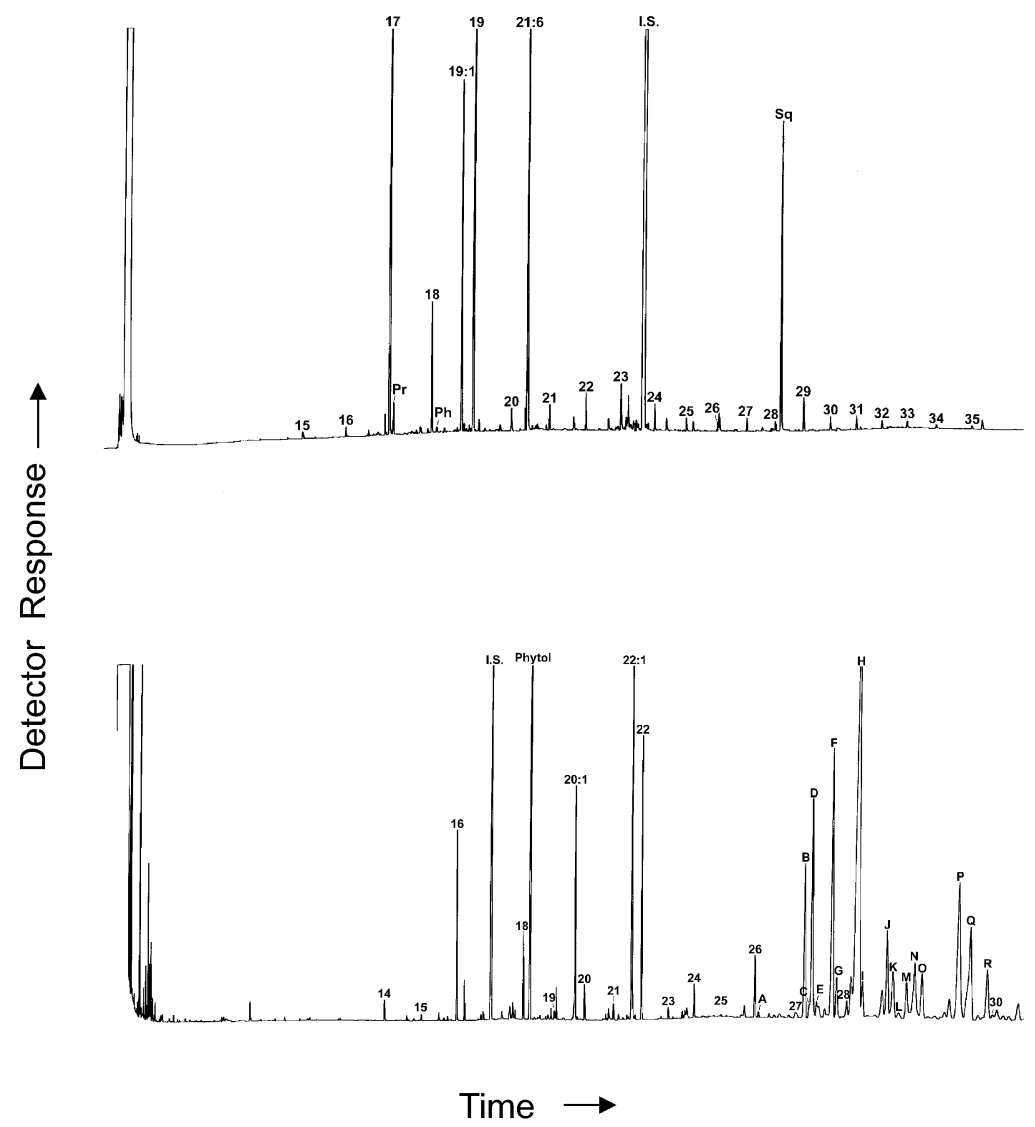

Fig. 2. (upper) Chromatogram of aliphatic hydrocarbons from the total suspended matter of station 14. (lower) Chromatogram of the alkanol/sterol fraction from the total suspended matter of station 22. Sterol identifications: (A) 24-nor-5 $\alpha$-cholest-22E-en-3 $\beta$-ol, (B)

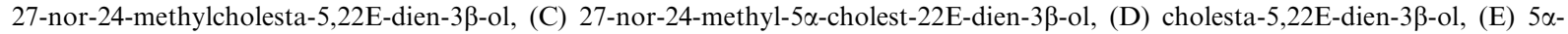

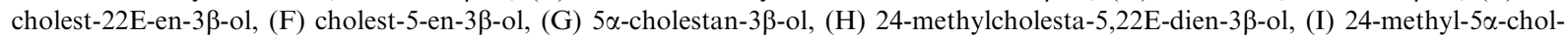

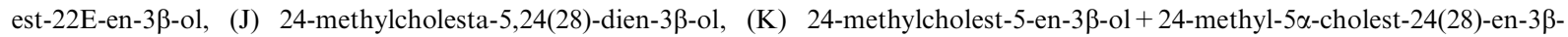

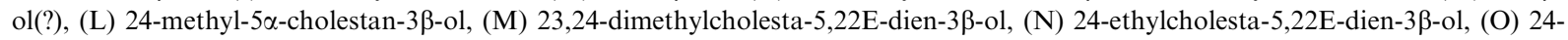

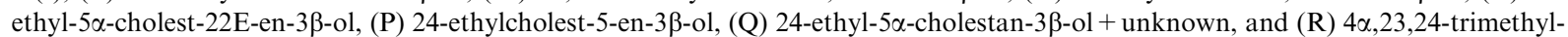
$5 \alpha$-cholest-22E-en-3 $\beta$-ol. 
Table 1

Concentrations $(\mu \mathrm{g} / \mathrm{g})$ of aliphatic hydrocarbons for total suspended matter

\begin{tabular}{|c|c|c|c|c|c|c|c|c|c|c|c|}
\hline \multirow[t]{2}{*}{ Compound } & \multicolumn{11}{|l|}{ Sample } \\
\hline & 10 & 12 & 14 & 16 & 18 & 20 & 22 & 24 & 32 & 34 & 36 \\
\hline$n-\mathrm{C}_{15}$ & - & - & 0.094 & 1.92 & - & - & - & - & - & - & 3.00 \\
\hline$n-\mathrm{C}_{16}$ & - & - & 0.171 & 1.74 & 0.540 & - & - & 0.635 & - & - & 1.93 \\
\hline$n-\mathrm{C}_{17}$ & 29.6 & 12.9 & 19.4 & 103. & 3.59 & - & 27.6 & 13.5 & 36.1 & 0.214 & 13.2 \\
\hline $\operatorname{Pr}$ & 3.25 & 2.63 & 0.692 & 4.01 & 0.519 & - & 1.35 & 4.17 & 5.36 & - & 4.06 \\
\hline$n-\mathrm{C}_{18}$ & 5.65 & 3.89 & 2.79 & 17.6 & 4.02 & 0.126 & 14.8 & 2.58 & 12.7 & 0.636 & 5.37 \\
\hline $\mathrm{Ph}$ & 0.503 & - & 0.176 & - & 0.757 & - & - & 0.461 & - & - & 1.20 \\
\hline$n-\mathrm{C}_{19: 1}$ & 38.9 & 11.2 & 7.52 & 54.0 & 4.48 & 0.318 & 45.2 & 12.7 & 50.6 & 4.52 & 7.49 \\
\hline$n-\mathrm{C}_{19}$ & 15.1 & 18.5 & 14.9 & 122. & 10.1 & 2.12 & 87.7 & 4.22 & 56.6 & 3.53 & 12.7 \\
\hline$n-\mathrm{C}_{20}$ & 0.996 & 0.639 & 0.506 & 1.94 & 3.35 & 0.315 & 2.54 & 0.539 & 1.66 & 0.448 & 1.64 \\
\hline$n-\mathrm{C}_{21: 6}$ & 7.91 & 23.3 & 16.6 & 30.9 & 17.0 & 1.58 & 56.7 & 48.5 & 104. & 8.49 & 43.3 \\
\hline$n-\mathrm{C}_{21}$ & 1.13 & 0.561 & 0.559 & 2.27 & 4.13 & 0.381 & 2.88 & 0.505 & 2.07 & 0.549 & 1.75 \\
\hline$n-\mathrm{C}_{22}$ & 1.08 & 0.498 & 0.776 & 1.46 & 4.09 & 0.412 & 2.20 & 0.653 & 1.96 & 0.583 & 1.98 \\
\hline$n-\mathrm{C}_{23}$ & 1.28 & 0.903 & 1.05 & 3.00 & 4.71 & 0.578 & 3.21 & 1.48 & 5.14 & 0.955 & 3.27 \\
\hline$n-\mathrm{C}_{24}$ & 0.577 & 0.654 & 0.559 & 1.56 & 3.13 & 0.426 & 2.20 & 1.37 & 3.43 & 0.651 & 2.32 \\
\hline$n-\mathrm{C}_{25}$ & 0.535 & 0.654 & 0.321 & 1.26 & 2.55 & 0.429 & 2.09 & 1.31 & 3.70 & 0.530 & 1.85 \\
\hline$n-\mathrm{C}_{26}$ & 0.461 & 0.420 & 0.142 & 0.709 & 2.92 & 0.358 & 2.14 & 0.940 & 2.61 & 0.579 & 1.24 \\
\hline$n-\mathrm{C}_{27}$ & 0.608 & 0.888 & 0.263 & 0.993 & 2.96 & 0.406 & 2.93 & 0.861 & 3.92 & 0.639 & 1.22 \\
\hline$n-\mathrm{C}_{28}$ & 0.598 & 0.748 & 0.198 & 0.709 & 2.79 & 0.398 & 1.92 & 0.609 & 3.27 & 1.01 & 0.693 \\
\hline $\mathrm{Sq}$ & 7.01 & 13.1 & 6.93 & 26.0 & 16.6 & 1.36 & 49.1 & 11.9 & 57.4 & 1.16 & 27.1 \\
\hline$n-\mathrm{C}_{29}$ & 1.05 & 1.62 & 0.757 & 1.84 & 4.28 & 0.942 & 7.61 & 0.766 & 7.48 & 1.79 & 2.91 \\
\hline$n-\mathrm{C}_{30}$ & 0.587 & 0.934 & 0.359 & 1.80 & 2.10 & 0.318 & 2.99 & 1.14 & 4.60 & 0.831 & 2.12 \\
\hline$n-\mathrm{C}_{31}$ & 0.818 & 0.966 & 0.345 & 1.28 & 3.13 & 0.693 & 4.85 & 0.557 & 4.46 & 1.09 & 2.12 \\
\hline$n-\mathrm{C}_{32}$ & 0.440 & 0.592 & 0.215 & 0.871 & 1.34 & 0.246 & 2.37 & 0.305 & 2.69 & 0.677 & 1.39 \\
\hline$n-\mathrm{C}_{33}$ & 0.493 & 0.452 & 0.166 & 0.669 & 1.25 & 0.366 & 2.59 & 0.287 & 2.56 & 0.613 & 0.774 \\
\hline$n-\mathrm{C}_{34}$ & 0.336 & - & 0.130 & 0.790 & 0.627 & 0.215 & 1.13 & 0.165 & 1.74 & 0.369 & 1.24 \\
\hline$n-\mathrm{C}_{35}$ & 0.419 & - & 0.137 & - & 1.32 & 0.200 & - & 0.315 & 1.52 & 0.560 & 0.342 \\
\hline$\% \mathrm{PDHC}$ & 90 & 89 & 91 & 94 & 55 & 45 & 87 & 88 & 86 & 61 & 80 \\
\hline CPI & 1.39 & 1.54 & 1.52 & 1.16 & 1.30 & 1.74 & 1.82 & 1.04 & 1.41 & 1.30 & 1.24 \\
\hline TSM & 4.81 & 4.06 & 13.1 & 1.66 & 1.39 & 12.1 & 1.49 & 8.00 & 0.128 & 5.68 & 2.40 \\
\hline
\end{tabular}

$\mathrm{Pr}=$ pristane $\mathrm{Ph}=$ phytane $\mathrm{Sq}=$ squalene

$\% \mathrm{PDHC}=\%$ planktonically-derived hydrocarbons

$$
\begin{aligned}
& =\%\left(n-\mathrm{C}_{17}+\mathrm{Pr}+n-\mathrm{C}_{18}+n-\mathrm{C}_{19: 1}+n-\mathrm{C}_{19}+n-\mathrm{C}_{21: 6}+\mathrm{Sq}\right) \\
& =\Sigma \mathrm{PDHC} / \Sigma \mathrm{HC}
\end{aligned}
$$

$\mathrm{CPI}=$ carbon preference index

$$
\begin{aligned}
& =\frac{1}{2}\left(\frac{n C_{25}+n C_{27}+n C_{29}+n C_{31}+n C_{33}}{n C_{24}+n C_{26}+n C_{28}+n C_{30}+n C_{32}}+\frac{n C_{25}+n C_{27}+n C_{29}+n C_{31}+n C_{33}}{n C_{26}+n C_{28}+n C_{30}+n C_{32}+n C_{34}}\right) \\
& =1.41 \text { (average) }
\end{aligned}
$$

$\mathrm{TSM}=$ total suspended matter $(\mathrm{mg} / \mathrm{l})$

$-=$ less than $0.05 \mu \mathrm{g} / \mathrm{g}$

Table 2

Spearman correlation coefficients for 7 planktonically-derived hydrocarbons of suspended matter. The coefficients were calculated using the rank values converted from lipid concentrations

\begin{tabular}{llllllll}
\hline & $\mathrm{C}_{17}$ & Pristane & $\mathrm{C}_{18}$ & $\mathrm{C}_{19: 1}$ & $\mathrm{C}_{19}$ & $\mathrm{C}_{21: 6}$ & Squalene \\
\hline $\mathrm{C}_{17}$ & 1 & & & & & \\
Pristane & $\mathbf{0 . 6 8 8}$ & 1 & & & & \\
$\mathrm{C}_{18}$ & $\mathbf{0 . 8 2 7}$ & 0.533 & 1 & & & \\
$\mathrm{C}_{19: 1}$ & $\mathbf{0 . 9 3 6}$ & $\mathbf{0 . 7 0 2}$ & $\mathbf{0 . 7 9 1}$ & 1 & & \\
$\mathrm{C}_{19}$ & $\mathbf{0 . 8 3 6}$ & 0.487 & $\mathbf{0 . 9 0 0}$ & $\mathbf{0 . 8 6 4}$ & 1 & \\
$\mathrm{C}_{21: 6}$ & 0.518 & $\mathbf{0 . 7 2 9}$ & 0.564 & $\mathbf{0 . 6 1 8}$ & 0.545 & $\mathbf{0 . 8 5 5}$ \\
Squalene & 0.573 & $\mathbf{0 . 6 5 1}$ & $\mathbf{0 . 8 0 9}$ & 0.591 & $\mathbf{0 . 7 0 0}$ & 1 \\
\hline
\end{tabular}

Numbers in boldface indicate values (except diagonal) at the significance level of $\alpha=0.05$ (two-tailed test). 
either diatoms or zooplankton. In the ECS, the phytoplankton was composed of 140 species, 104 of which belong to Bacillariophyceae (Wu et al., 2000). In summer and winter, copepods dominate the zooplankton community (Liu and He, 1990). Diatoms and copepods are both likely sources of $n$ - $\mathrm{C}_{21: 6}$, pristane and squalene.
On the other hand, a calculation of the carbon preference index (CPI) in the range of $\mathrm{C}_{25}-\mathrm{C}_{33}$ does not show any consistent geographic distribution for these minor $n$-alkanes which suggests that the terrigenous input from the Changjiang River does not imprint the TSM distribution.

Table 3

Concentrations $(\mu \mathrm{g} / \mathrm{g})$ of alkanols and sterols for total suspended matter

\begin{tabular}{|c|c|c|c|c|c|c|c|c|c|c|c|}
\hline \multirow[t]{2}{*}{ Compound } & \multicolumn{11}{|l|}{ Sample } \\
\hline & 10 & 12 & 14 & 16 & 18 & 20 & 22 & 24 & 32 & 34 & 36 \\
\hline \multicolumn{12}{|l|}{ Alkanols } \\
\hline$n-\mathrm{C}_{14} \mathrm{OH}$ & 1.17 & 2.29 & 0.339 & 2.51 & 4.82 & 0.497 & 7.00 & 3.17 & 6.08 & 1.30 & 4.59 \\
\hline$n-\mathrm{C}_{15} \mathrm{OH}$ & 0.317 & 0.512 & 0.142 & 1.43 & 2.13 & 0.263 & 2.11 & 0.662 & 2.33 & 0.424 & 1.17 \\
\hline$n-\mathrm{C}_{16} \mathrm{OH}$ & 5.68 & 13.7 & 1.37 & 19.2 & 16.2 & 6.97 & 70.2 & 21.6 & 36.7 & 12.2 & 34.4 \\
\hline$n-\mathrm{C}_{18} \mathrm{OH}$ & 8.92 & 19.2 & 2.97 & 39.1 & 28.0 & 5.53 & 34.1 & 9.42 & 32.3 & 13.0 & 17.8 \\
\hline phytol & 112 & 81.5 & 67.6 & 166 & 137 & 20.9 & 364 & 184 & 566 & 48.9 & 126 \\
\hline$n-\mathrm{C}_{19} \mathrm{OH}$ & 1.07 & 2.08 & 0.442 & 2.29 & 7.11 & 0.289 & 2.73 & 1.22 & 5.63 & 1.15 & 1.66 \\
\hline$n-\mathrm{C}_{20: 1} \mathrm{OH}$ & 0.947 & - & - & - & - & 11.5 & 93.8 & 31.3 & 11.5 & 9.86 & 48.6 \\
\hline$n-\mathrm{C}_{20} \mathrm{OH}$ & 2.53 & 3.44 & 0.881 & 8.92 & 12.9 & 1.92 & 13.8 & 3.57 & 10.3 & 3.17 & 7.30 \\
\hline$n-\mathrm{C}_{21} \mathrm{OH}$ & 1.08 & 1.85 & 0.407 & 3.06 & 4.01 & 0.740 & 7.72 & 2.69 & 3.18 & 0.730 & 3.47 \\
\hline$n-\mathrm{C}_{22: 1} \mathrm{OH}$ & 2.86 & - & - & - & 5.18 & 25.1 & 217 & 73.2 & 28.5 & 19.8 & 105 \\
\hline$n-\mathrm{C}_{22} \mathrm{OH}$ & 30.2 & 42.6 & 9.16 & 79.6 & 166 & 10.6 & 108 & 26.4 & 46.3 & 15.4 & 52.3 \\
\hline$n-\mathrm{C}_{23} \mathrm{OH}$ & 1.22 & 1.60 & 0.413 & 3.43 & 6.60 & 0.493 & 4.32 & 1.14 & 2.27 & 0.701 & 1.92 \\
\hline$n-\mathrm{C}_{24} \mathrm{OH}$ & 3.58 & 4.32 & 1.18 & 9.41 & 19.4 & 1.42 & 12.4 & 2.97 & 7.27 & 2.04 & 5.30 \\
\hline$n-\mathrm{C}_{25} \mathrm{OH}$ & 0.420 & - & 0.161 & - & 2.26 & 0.179 & - & 0.225 & 1.70 & 0.226 & - \\
\hline$n-\mathrm{C}_{26} \mathrm{OH}$ & 15.1 & 10.1 & 3.54 & 25.1 & 12.5 & 0.390 & 41.3 & 10.7 & 31.5 & 0.549 & 10.3 \\
\hline$n-\mathrm{C}_{27} \mathrm{OH}$ & 0.488 & - & 0.558 & - & 8.15 & - & 3.76 & 0.912 & 4.20 & - & 0.481 \\
\hline$n-\mathrm{C}_{28} \mathrm{OH}$ & 3.47 & 7.77 & 1.20 & 5.49 & 52.5 & 0.594 & 16.2 & 1.29 & 21.0 & 1.98 & 7.16 \\
\hline \multicolumn{12}{|l|}{ Sterols } \\
\hline A & 0.760 & 0.900 & 0.300 & 1.77 & 1.09 & 1.16 & 3.76 & 0.687 & 2.46 & 0.571 & 1.07 \\
\hline B & 28.2 & 43.1 & 9.20 & 39.8 & 21.8 & 5.34 & 151 & 43.9 & 91.1 & 31.0 & 44.4 \\
\hline $\mathrm{C}$ & 1.50 & - & 0.436 & - & 2.20 & 0.445 & 10.2 & 1.80 & - & 1.62 & 3.63 \\
\hline $\mathrm{D}$ & 36.4 & 60.7 & 21.3 & 106 & 45.7 & 9.28 & 202 & 22.2 & 138 & 27.1 & 51.2 \\
\hline E & 3.92 & 3.14 & 0.852 & 8.85 & 18.9 & 1.77 & 8.03 & 1.86 & 23.4 & 7.64 & 3.61 \\
\hline $\mathrm{F}$ & 40.3 & 97.6 & 44.7 & 101 & 101 & 20.5 & 295 & 50.8 & 281 & 50.3 & 78.3 \\
\hline G & 4.77 & 10.2 & 4.15 & 24.5 & 28.4 & 4.49 & 40.4 & 4.68 & 46.7 & 8.87 & 11.2 \\
\hline $\mathrm{H}$ & 256 & 226 & 102 & 552 & 135 & 35.1 & 863 & 121 & 808 & 122 & 176 \\
\hline I & 4.08 & 8.54 & 2.82 & 15.4 & 19.2 & 3.36 & 30.3 & 3.55 & 28.9 & 5.00 & 8.92 \\
\hline $\mathrm{J}$ & 18.0 & 47.2 & 21.2 & 68.0 & 54.2 & 9.17 & 101 & 36.8 & 127 & 18.0 & 26.4 \\
\hline K & 8.53 & 21.4 & 9.85 & 55.0 & 40.3 & 7.61 & 62.7 & 6.03 & 41.8 & 10.9 & 5.86 \\
\hline $\mathrm{L}$ & 1.51 & 2.78 & 0.933 & 7.15 & 9.27 & 1.22 & 7.72 & 0.954 & 3.76 & 2.16 & - \\
\hline M & 6.03 & 17.6 & 3.16 & 22.6 & 13.7 & 2.59 & 38.4 & 4.60 & 29.8 & 8.81 & 7.74 \\
\hline $\mathrm{N}$ & 14.1 & 23.6 & 11.2 & 76.6 & 63.4 & 5.19 & 87.0 & 11.1 & 109 & 30.9 & 21.5 \\
\hline $\mathrm{O}$ & 15.3 & 13.6 & 4.02 & 41.3 & 30.8 & 6.21 & 55.9 & 11.1 & 56.2 & 10.7 & 10.6 \\
\hline$P$ & 27.9 & 76.0 & 32.9 & 102 & 65.5 & 11.0 & 260 & 46.8 & 221 & 65.3 & 83.7 \\
\hline Q & 37.0 & 44.5 & 49.2 & 91.3 & 68.0 & 15.8 & 183 & 36.4 & 188 & 30.0 & 59.7 \\
\hline $\mathrm{R}$ & 19.3 & 16.5 & 7.44 & 47.3 & 67.2 & 11.4 & 72.0 & 44.2 & 92.3 & 25.8 & 20.4 \\
\hline $\mathrm{B}+\mathrm{D}+\mathrm{F}+\mathrm{H}$ & 360.9 & 427.4 & 177.2 & 798.8 & 303.5 & 70.2 & 1511 & 237.9 & 1318 & 230.4 & 349.9 \\
\hline$\Sigma \mathrm{A} \sim \mathrm{R}$ & 523.6 & 713.4 & 325.7 & 1360 & 785.6 & 151.6 & 2471 & 448.5 & 2288 & 448.7 & 632.3 \\
\hline$\%$ major sterols & 69 & 60 & 54 & 59 & 39 & 46 & 61 & 53 & 58 & 51 & 55 \\
\hline TSM & 4.81 & 4.06 & 13.1 & 1.66 & 1.39 & 12.1 & 1.49 & 8.00 & 0.128 & 5.68 & 2.40 \\
\hline
\end{tabular}

Sterol identifications: (A) 24-nor-5 $\alpha$-cholest-22E-en-3 $\beta$-ol, (B) 27-nor-24-methylcholesta-5,22E-dien-3 $\beta$-ol, (C) 27-nor-24-methyl-5 $\alpha$ -

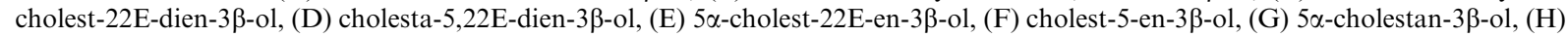

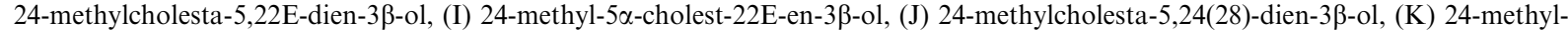

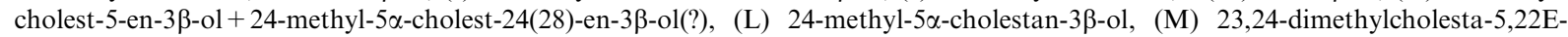

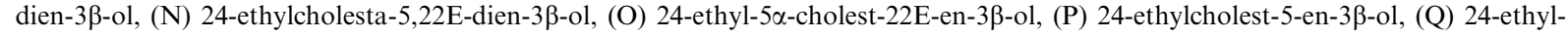

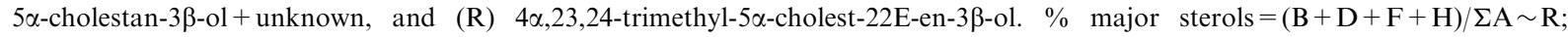
$\mathrm{TSM}=$ total suspended matter $(\mathrm{mg} / \mathrm{l}) ;-=$ less than $0.1 \mu \mathrm{g} / \mathrm{g}$. 


\subsubsection{Fatty alcohols}

The distribution of $n$-alkanols observed in suspended matter was similar among all stations, and was dominated by shorter chain $\left(<\mathrm{C}_{22}\right)$ alcohols with phytol being the most significant constituent (Fig. 2, Table 3). This distribution reflects a major contribution from marine sources. In contrast, saturated alcohols from higher plant waxes $\left(>\mathrm{C}_{22}\right)$ were present in a minor proportion, indicating that terrigenous alcohols played a minor role. Among the shorter chain alcohols, monounsaturated $n$-alkenols $\mathrm{C}_{20: 1}$ and $\mathrm{C}_{22: 1}$ can originate from marine zooplankton especially calanoid copepods (Sargent and Lee, 1975; Saito and Kotani, 2000). Both $\mathrm{C}_{20: 1}$ and $\mathrm{C}_{22: 1} n$-alkenols were not detected at the stations close to land (stations 12, 14, and 16) and were present at much higher concentrations at the stations farther offshore (stations 20, 22, 24, 42, 34 and 36). This spatial distribution suggests a cross-shelf gradient of zooplankton, with higher abundances offshore.

Spearman correlation coefficients showed that hexadecan-1-ol and $\mathrm{C}_{20: 1}$ and $\mathrm{C}_{22: 1}$ n-alkenols were correlated (Table 4), their most likely source being copepods (Sargent and Lee, 1975). Copepods are the most dominant zooplankton in the Changjiang River and its adjacent waters (Liu and He, 1990).

\subsubsection{Sterols}

All GC traces of sterols (Fig. 2) in the suspended matter were very similar. The major feature was the dominance of 24-methylcholesta-5,22E-dien-3 $\beta$-ol (brassicasterol-diatomsterol), cholest-5-en-3 $\beta$-ol (cholesterol), cholesta-5,22E-dien-3 $\beta$-ol (22-dehydrocholesterol), and 27 -nor-24-methylcholesta-5,22E-dien-3 $\beta$-ol. They accounted for an average of $55 \%$ of total sterols (Table 3 ), which ranks in the upper proportion of marine sterol distributions when compared to particles sampled closer to the shore $(36,44$ and $57 \%$ as calculated from Tian et al., 1992; Sicre et al., 1993b, 1994, respectively). In the suspended matter, sterols common to diatoms, dinoflagellates and zooplankton made up the major proportion of the sterol fraction (Goad and Withers, 1982; Volkman, 1986) (Table 3). The biomarkers for a given species of plankton are not always specific enough to identify a single source (Volkman, 1986; Wakeham, 1995). Stations 22 and 32 were distinguished from other stations by their high concentrations of 24-methyl-cholesta-5,24(28)-dien-3 $\beta$-ol and $4 \alpha, 23,24$ trimethyl-5 $\alpha$-cholest-22E-en-3 $\beta$-ol (dinosterol), but a major contribution from dinoflagellates (Volkman, 1986) could not be conclusive.

Table 5 shows the Spearman correlations of the 18 sterols quantified. Almost all sterols were significantly correlated except 27-nor-24-methyl-5 $\alpha$-cholest-22E-dien$3 \beta$-ol. This sterol was the fourth most abundant and was only correlated with 9 sterols of the 17 sterols. This compound was found in suspended particles of the

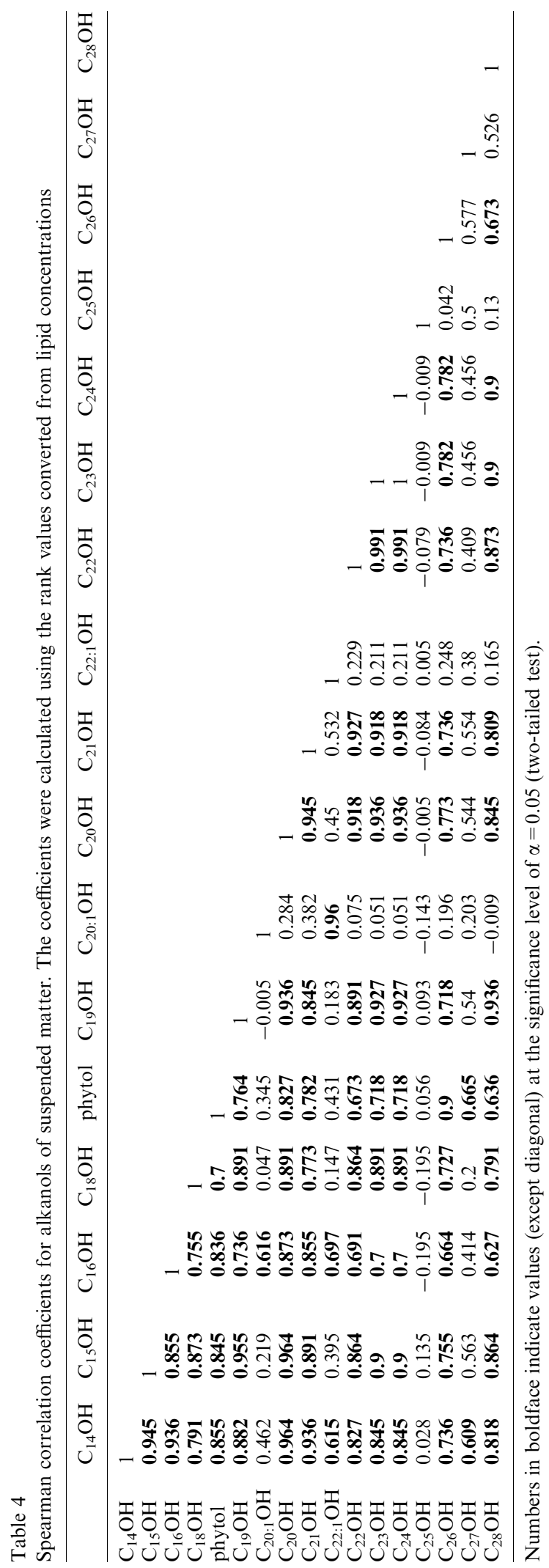


Changjiang Estuary by Sicre et al. (1994), but not by Tian et al. (1992) nor Sicre et al. (1993b). This might suggest the sterol from a group of marine plankton only blooming in certain time and at some locations. The three common sterols of higher plant origin, 24-methylcholest-5-en-3 $\beta$-ol (campesterol), 24-ethylcholesta-5,22Edien-3 $\beta$-ol (stigmasterol) and 24-ethylcholest-5-en-3 $\beta$-ol (sitosterol), were correlated with most sterols of marine origin, possibly implying predominantly similar marine sources. As examples, some dinoflagellates and diatoms contain 24-methylcholest-5-en-3ß-ol (campesterol) (Volkman, 1986). Among the 14 species of marine diatoms from the CSIRO Culture Collection of Microalgae (sources mainly from Australian waters), most of the centric species have 24-methylcholesta-5,24(28)-dien-3 $\beta$ ol (24-methylenecholesterol) and 24-methylcholest-5-en$3 \beta$-ol (campesterol) as two of their major sterols (Barrett et al., 1995). Four species of marine coccolithophorids (from Plymouth Culture Collection, UK) have been reported to contain 24-ethylcholesta-5,22E-dien-3 $\beta$-ol (stigmasterol) as the major component (Volkman et al., 1981). 24-Ethylcholest-5-en-3ß-ol (sitosterol) has been found in diatoms, prymnesiophyceae, chlorophyceae, and cyanobacteria (Volkman, 1986). 24-Ethylcholesta$5,22 \mathrm{E}$-dien-3 $\beta$-ol (stigmasterol) is the principal sterol in diatom Amphora coffaeformis, while 24-ethylcholest-5- en-3 $\beta$-ol (sitosterol) is the major sterol in diatom Navicula pelliculosa (Gladu et al., 1991). The present results suggest that most sterols are derived from marine sources. However, in a previous study by Sicre et al. (1994) on sterols from the suspended particles of the Changjiang Estuary, it was concluded from factor analysis that 24 -ethylcholesta-5,22-dien-3 $\beta$-ol was probably $\beta$-sitosterol, a tracer of terrigenous inputs.

\subsection{Comparison of TSM with sediment}

\subsubsection{Degradation of lipids}

The sedimentary aliphatic hydrocarbon composition in the nine samples studied was quite similar. The unsaturated hydrocarbons $n-\mathrm{C}_{19: 1}$ and $n-\mathrm{C}_{21: 6}$ were in trace to minor quantities, but squalene was a major constituent (Table 6). The difference between suspended particles and sediments could be attributed to the easy degradation of labile hydrocarbons of algal $\left(n-\mathrm{C}_{19: 1}\right.$ and $n-\mathrm{C}_{21: 6}$ ) origin relative to terrigenous components (Meyers et al. 1984; Gagosian and Peltzer; 1986). In this respect, a comparison of aliphatic hydrocarbons in plankton, sediment trap particulates and sediments from Dabob Bay indicates that most pronounced decreases have been found for pristane and two unsaturated compounds (Prahl et al., 1980). Furthermore, in

Table 5

Spearman correlation coefficients for sterols of suspended matter. The coefficients were calculated using the rank values converted from lipid concentrations

\begin{tabular}{|c|c|c|c|c|c|c|c|c|c|c|c|c|c|c|c|c|c|c|}
\hline & A & B & $\mathrm{C}$ & $\mathrm{D}$ & $\mathrm{E}$ & $\mathrm{F}$ & G & $\mathrm{H}$ & I & $\mathbf{J}$ & $\mathrm{K}$ & $\mathrm{L}$ & M & $\mathrm{N}$ & $\mathrm{O}$ & $\mathrm{P}$ & Q & $\mathrm{R}$ \\
\hline A & 1 & & & & & & & & & & & & & & & & & \\
\hline B & 0.445 & 1 & & & & & & & & & & & & & & & & \\
\hline $\mathrm{C}$ & 0.018 & 0.220 & 1 & & & & & & & & & & & & & & & \\
\hline D & 0.700 & 0.773 & -0.018 & 1 & & & & & & & & & & & & & & \\
\hline E & 0.609 & 0.418 & 0.028 & 0.736 & 1 & & & & & & & & & & & & & \\
\hline $\mathrm{F}$ & 0.674 & 0.724 & 0.087 & 0.897 & 0.743 & 1 & & & & & & & & & & & & \\
\hline G & 0.755 & 0.664 & 0.110 & 0.909 & 0.900 & 0.916 & 1 & & & & & & & & & & & \\
\hline $\mathrm{H}$ & 0.645 & 0.709 & -0.046 & 0.945 & 0.727 & 0.756 & 0.818 & 1 & & & & & & & & & & \\
\hline I & 0.764 & 0.673 & 0.193 & 0.918 & 0.873 & 0.925 & 0.991 & 0.827 & 1 & & & & & & & & & \\
\hline $\mathbf{J}$ & 0.647 & 0.688 & -0.078 & 0.852 & 0.688 & 0.963 & 0.843 & 0.738 & 0.834 & 1 & & & & & & & & \\
\hline K & 0.591 & 0.291 & -0.220 & 0.718 & 0.700 & 0.756 & 0.700 & 0.682 & 0.718 & 0.738 & 1 & & & & & & & \\
\hline $\mathrm{L}$ & 0.618 & 0.191 & -0.046 & 0.636 & 0.791 & 0.711 & 0.736 & 0.600 & 0.755 & 0.661 & 0.891 & 1 & & & & & & \\
\hline M & 0.655 & 0.691 & -0.046 & 0.955 & 0.809 & 0.920 & 0.918 & 0.882 & 0.927 & 0.856 & 0.845 & 0.782 & 1 & & & & & \\
\hline $\mathrm{N}$ & 0.618 & 0.573 & -0.055 & 0.882 & 0.900 & 0.879 & 0.927 & 0.818 & 0.918 & 0.815 & 0.855 & 0.782 & 0.955 & 1 & & & & \\
\hline $\mathrm{O}$ & 0.700 & 0.573 & -0.073 & 0.836 & 0.864 & 0.806 & 0.855 & 0.873 & 0.845 & 0.825 & 0.773 & 0.818 & 0.864 & 0.836 & 1 & & & \\
\hline$P$ & 0.655 & 0.818 & 0.064 & 0.936 & 0.682 & 0.943 & 0.891 & 0.800 & 0.900 & 0.879 & 0.673 & 0.545 & 0.918 & 0.873 & 0.709 & 1 & & \\
\hline Q & 0.645 & 0.573 & -0.028 & 0.836 & 0.691 & 0.870 & 0.827 & 0.764 & 0.818 & 0.893 & 0.691 & 0.545 & 0.782 & 0.818 & 0.727 & 0.845 & 1 & \\
\hline $\mathrm{R}$ & 0.636 & 0.645 & 0.229 & 0.709 & 0.873 & 0.834 & 0.864 & 0.664 & 0.855 & 0.802 & 0.618 & 0.691 & 0.773 & 0.809 & 0.855 & 0.745 & 0.691 & 1 \\
\hline
\end{tabular}

Numbers in boldface indicate values (except diagonal) at the significance level of $\alpha=0.05$ (two-tailed test). Sterol identifications: (A) 24-nor$5 \alpha$-cholest-22E-en-3 $\beta$-ol, (B) 27-nor-24-methylcholesta-5,22E-dien-3 $\beta$-ol, (C) 27-nor-24-methyl-5 $\alpha$-cholest-22E-dien-3 $\beta$-ol, (D) cholesta-

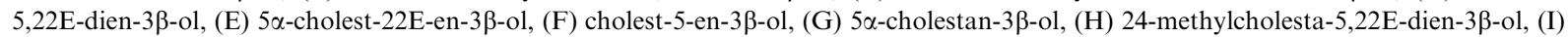

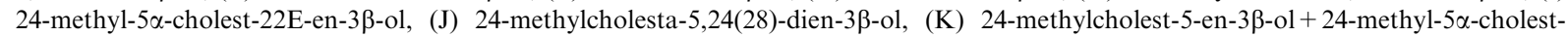

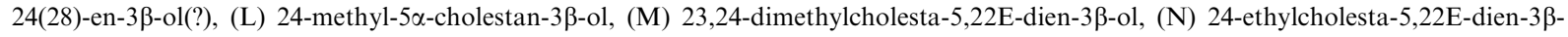

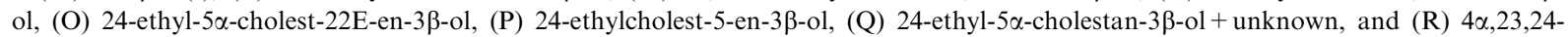
trimethyl-5 $\alpha$-cholest-22E-en-3 $\beta$-ol. 
a deltaic environment, labile hydrocarbons of both algal $\left(\mathrm{C}_{15: 1}, \mathrm{C}_{17: 1}\right.$ and $\mathrm{C}_{21: 6}$ etc. $)$ origin have not been found in the underlying sediments (water depths $<20 \mathrm{~m}$ ) due to prevailing oxic conditions (Albaiges et al., 1984). Assuming that the PDHCs in sediments were deposited from the overlying suspended matter. Their degradation was assessed by the ratio between normalized concentration in TSM and sediments. In both types of samples PDHC concentrations were normalized to $n-C_{23}$. The ratio values vary over 2 orders of magnitude, the highest corresponding to the most labile compounds (Table 7). By order of decreasing degradation, PDHCs were ranked as follows: $n-\mathrm{C}_{21: 6}>n-\mathrm{C}_{19: 1}, n-\mathrm{C}_{19}$ and $n-\mathrm{C}_{17}>$ $n-\mathrm{C}_{18}$, pristane and squalene. This result is similar to that in Albaiges et al. (1984). In the case of squalene, the hypothesis that this compound was mainly from the water column is not valid, as halophilic bacteria are an important source of sedimentary squalene under non-aerated conditions (Tornabene, 1978), which distorts the calculated ratio (Table 7).

Similar ratios may be calculated for the linear alcohols. In this case, $n$-eicosan-1-ol was used as a reference compound since it is not substantially enhanced by inputs of land or marine plants. Both $\mathrm{C}_{20: 1}$ and $\mathrm{C}_{22: 1} n^{-}$ alkenols degrade faster than phytol i.e., by factors of 3 4 (Table 7). Compared to $n$-alkanols, phytol is relatively labile in sediment. Extractable phytol in sediments has been shown to be highly reactive in studies from other locations, partly as a result of its conversion to bound phytol (Jeng et al., 1997; Sun et al., 1998).

Application of the same approach to the sterols showed lower degradation values than PDHCs. These low values indicate that sterols are relatively resistant to degradation, which is in agreement with the results

Table 6

Concentrations (ng/g) of aliphatic hydrocarbons for bottom sediments

\begin{tabular}{|c|c|c|c|c|c|c|c|c|c|}
\hline \multirow[t]{2}{*}{ Compound } & \multicolumn{9}{|c|}{ Sample } \\
\hline & 10 & 14 & 18 & 20 & 22 & 24 & 32 & 34 & 36 \\
\hline$n-\mathrm{C}_{15}$ & - & - & - & - & - & - & - & 5 & 6 \\
\hline$n-\mathrm{C}_{16}$ & 2 & - & 5 & 4 & 6 & 3 & 3 & 15 & 18 \\
\hline$n-\mathrm{C}_{17}$ & 4 & 7 & 15 & 18 & 18 & 24 & 14 & 25 & 37 \\
\hline Pristane & 6 & 3 & 10 & 11 & 9 & 32 & 9 & 12 & 21 \\
\hline$n-\mathrm{C}_{18}$ & 4 & 12 & 15 & 18 & 19 & 13 & 17 & 20 & 30 \\
\hline Phytane & 1 & 4 & 5 & 6 & 7 & 5 & 6 & 6 & 11 \\
\hline$n-\mathrm{C}_{19: 1}$ & 1 & 5 & 2 & 4 & 5 & 18 & 14 & 3 & 5 \\
\hline$n-\mathrm{C}_{19}$ & 3 & 13 & 11 & 18 & 13 & 16 & 13 & 16 & 24 \\
\hline$n-\mathrm{C}_{20}$ & 2 & 13 & 9 & 14 & 10 & 8 & 10 & 12 & 19 \\
\hline$n-\mathrm{C}_{21: 6}$ & 1 & 3 & 3 & 3 & 2 & - & 4 & 3 & 4 \\
\hline$n-\mathrm{C}_{21}$ & 8 & 20 & 11 & 24 & 18 & 12 & 16 & 18 & 26 \\
\hline$n-\mathrm{C}_{22}$ & 3 & 18 & 8 & 17 & 12 & 8 & 13 & 14 & 21 \\
\hline$n-\mathrm{C}_{23}$ & 5 & 31 & 17 & 33 & 22 & 14 & 26 & 32 & 39 \\
\hline$n-\mathrm{C}_{24}$ & 3 & 21 & 11 & 21 & 14 & 10 & 19 & 20 & 21 \\
\hline$n-\mathrm{C}_{25}$ & 5 & 40 & 18 & 43 & 27 & 15 & 33 & 42 & 40 \\
\hline$n-\mathrm{C}_{26}$ & 3 & 29 & 11 & 27 & 18 & 10 & 21 & 24 & 22 \\
\hline$n-\mathrm{C}_{27}$ & 7 & 65 & 29 & 69 & 43 & 22 & 51 & 74 & 56 \\
\hline$n-\mathrm{C}_{28}$ & 7 & 33 & 16 & 33 & 18 & 14 & 25 & 33 & 27 \\
\hline Squalene & - & - & 115 & 154 & 105 & 150 & 144 & 105 & 198 \\
\hline$n-\mathrm{C}_{29}$ & 15 & 136 & 65 & 140 & 94 & 48 & 106 & 167 & 118 \\
\hline$n-\mathrm{C}_{30}$ & 13 & 60 & 44 & 81 & 45 & 45 & 50 & 62 & 68 \\
\hline$n-\mathrm{C}_{31}$ & 14 & 167 & 77 & 171 & 112 & 51 & 127 & 217 & 136 \\
\hline$n-\mathrm{C}_{32}$ & 2 & 21 & 9 & 24 & 15 & 9 & 17 & 22 & 23 \\
\hline Diploptene & 13 & 58 & 32 & 70 & 47 & 28 & 53 & 81 & 57 \\
\hline$n-\mathrm{C}_{33}$ & 6 & 80 & 39 & 75 & 46 & 19 & 52 & 89 & 65 \\
\hline$n-\mathrm{C}_{34}$ & 1 & 9 & 7 & 13 & 7 & 5 & 8 & 13 & 13 \\
\hline$n-\mathrm{C}_{35}$ & 6 & 48 & 32 & 52 & 36 & 27 & 33 & 42 & 70 \\
\hline CPI & 2.74 & 3.10 & 2.57 & 2.74 & 3.03 & 1.82 & 2.93 & 3.74 & 2.65 \\
\hline
\end{tabular}

$\mathrm{CPI}=$ carbon preference index

$$
=\frac{1}{2}\left(\frac{n C_{25}+n C_{27}+n C_{29}+n C_{31}+n C_{33}}{n C_{24}+n C_{26}+n C_{28}+n C_{30}+n C_{32}}+\frac{n C_{25}+n C_{27}+n C_{29}+n C_{31}+n C_{33}}{n C_{26}+n C_{28}+n C_{30}+n C_{32}+n C_{34}}\right)=2.81 \text { (average) }
$$

$-=$ less than $1 \mathrm{ng} / \mathrm{g}$ 
reported by Saliot et al. (1991) and Quemeneur and Marty (1992). It is noted that the three most readily degraded sterols were 24-methylcholesta-5,22E-dien-3 $\beta$ ol, 27-nor-24-methylcholest-5,22-dien-3 $\beta$-ol and 24methylcholesta-5,24(28)-dien-3 $\beta$-ol. These diatom and dinoflagellate derived sterols appear to be relatively more labile.

The above reported comparisons involve the assumption that the predominant lipid sources for the sediments are only from the TSM, and that the concentrations sampled at 5 meter depth are representative of the water column composition. Otherwise, the ratios cannot be interpreted in terms of degradation processes.

\subsubsection{Carbon preference index of $n$-alkanes}

Carbon preference indices (CPIs) of suspended matter varied between 1.04 and 1.82 with an average of 1.41 (Table 1), and those of sediment ranged between 1.82 and 3.74 with a mean of 2.81 (Table 6). The lower CPIs in suspended matter than in sediment are similar to the observations reported in Dabob Bay (Washington) in which $n$-alkane $\mathrm{CPI}_{20-34}$ averaged 2.52 in sediment traps and 3.99 in surface sediments (Prahl et al., 1980).

\subsubsection{Sterol concentration difference between TSM and sediments}

Despite the general similarity in the sterol distribution of suspended matter (Fig. 2) and sediments (Fig. 3), there are significant differences in the relative proportions of sterols. In TSM 24-methylcholesta-5,22E-dien$3 \beta$-ol was the most abundant constituent followed by cholest-5-en-3 $\beta$-ol (Table 3), but this order is reversed in the sediments (Table 8), as currently observed in other coastal marine sediments (Harvey, 1994; Mudge and Norris, 1997). The predominance of cholest-5-en-3 $\beta$-ol in the sediments of the ECS suggests the superimposition of meiofaunal lipids (Goad, 1978). In this respect, the studied sediments contained shell fragments and cholesterol is usually the dominant sterol of mollusks (Ballantine et al., 1983 and references therein). At station 18, the sterol profiles in TSM and sediment were remarkably similar, which may reflect a major contribution from resuspended sediment (Tables 3 and 8).

Table 7

Ratios of planktonically-derived hydrocarbons normalized to $n-\mathrm{C}_{23}$ for TSM to those for sediments as well as ratios of alkenols, phytol and sterols normalized to $n-\mathrm{C}_{20} \mathrm{OH}$ for TSM to those for sediments

Compound Ratio

Hydrocarbons

Average $\left(n-\mathrm{C}_{17} / n-\mathrm{C}_{23}\right)_{\mathrm{TSM}} /$ average $\left(n-\mathrm{C}_{17} / n-\mathrm{C}_{23}\right)_{\text {sediment }}$

Average (pristane $\left./ n-\mathrm{C}_{23}\right)_{\mathrm{TSM}} /$ average (pristane $\left./ n-\mathrm{C}_{23}\right)_{\text {sediment }}$

Ratio

Average $\left(n-\mathrm{C}_{18} / n-\mathrm{C}_{23}\right)_{\mathrm{TSM}} /$ average $\left(n-\mathrm{C}_{18} / n-\mathrm{C}_{23}\right)_{\text {sediment }}$

Average $\left(n-\mathrm{C}_{19: 1} / n-\mathrm{C}_{23}\right)_{\mathrm{TSM}} /$ average $\left(n-\mathrm{C}_{19: 1} / n-\mathrm{C}_{23}\right)_{\text {sediment }}$
Average $\left(n-\mathrm{C}_{19} / n-\mathrm{C}_{23}\right)_{\mathrm{TSM}} /$ average $\left(n-\mathrm{C}_{19} / n-\mathrm{C}_{23}\right)_{\text {sediment }}$

Average $\left(n-\mathrm{C}_{21: 6} / n-\mathrm{C}_{23}\right)_{\text {TSM }} /$ average $\left(n-\mathrm{C}_{21: 6} / n-\mathrm{C}_{23}\right)_{\text {sediment }}$

Average (squalene $\left./ n-\mathrm{C}_{23}\right)_{\mathrm{TSM}} /$ average (squalene $/ n-\mathrm{C}_{23}$ ) sediment

Alcohols

Average $\left(\mathrm{C}_{20: 1} \mathrm{OH} / n-\mathrm{C}_{20} \mathrm{OH}\right)_{\mathrm{TSM}} /$ average $\left(\mathrm{C}_{20: 1} \mathrm{OH} / n-\mathrm{C}_{20} \mathrm{OH}\right)_{\text {sediment }}$

Average (phytol $\left./ n-\mathrm{C}_{20} \mathrm{OH}\right)_{\mathrm{TSM}} /$ average $\left(\mathrm{phytol} / n-\mathrm{C}_{20} \mathrm{OH}\right)_{\text {sediment }}$

Sterols

Average (27-nor-24-methylcholest-5,22-dien-3 $\beta$-ol $/ n$ - $\left.\mathrm{C}_{20} \mathrm{OH}\right)_{\mathrm{TSM}} /$ average (27-nor-24-methylcholest-

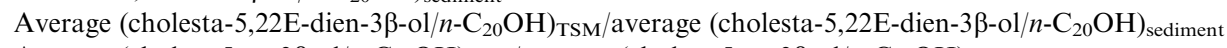

Average (cholest-5-en-3 $\beta$-ol $/ n$ - $\left.\mathrm{C}_{20} \mathrm{OH}\right)_{\mathrm{TSM}} /$ average (cholest-5-en-3 $\beta$-ol $/ n$ - $\mathrm{C}_{20} \mathrm{OH}$ ) sediment

Average (24-methylcholesta-5,22E-dien-3 $\beta$-ol $\left./ n-\mathrm{C}_{20} \mathrm{OH}\right)_{\mathrm{TSM}} /$ average (24-methylcholesta-5,22E-dien-3 $\beta$-ol/ $\left.n-\mathrm{C}_{20} \mathrm{OH}\right)_{\text {sediment }}$

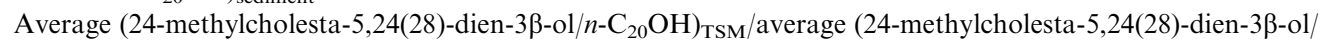
$\left.n-\mathrm{C}_{20} \mathrm{OH}\right)_{\text {sediment }}$

Average (24-methylcholest-5-en-3 $\beta$-ol $\left./ n-\mathrm{C}_{20} \mathrm{OH}\right)_{\text {TSM }} /$ average (24-methylcholest-5-en-3 $\beta$-ol $\left./ n-\mathrm{C}_{20} \mathrm{OH}\right)_{\text {sediment }}$

Average (24-ethylcholesta-5,22E-dien-3 $\beta$-ol $\left./ n-\mathrm{C}_{20} \mathrm{OH}\right)_{\mathrm{TSM}} /$ average (24-ethylcholesta-5,22E-dien-3 $\beta$-ol/ $\left.n-\mathrm{C}_{20} \mathrm{OH}\right)_{\text {sediment }}$

Average (24-ethylcholest-5-en-3 $\beta$-ol $\left./ n-\mathrm{C}_{20} \mathrm{OH}\right)_{\text {TSM }} /$ average (24-ethylcholest-5-en-3 $\beta$-ol $\left./ n-\mathrm{C}_{20} \mathrm{OH}\right)_{\text {sediment }}$

Average $\left(4 \alpha, 23,24 \text {-trimethyl-5 } \alpha \text {-cholest-22E-en-3 } \beta \text {-ol } / n \text { - } \mathrm{C}_{20} \mathrm{OH}\right)_{\mathrm{TSM}} /$ average $(4 \alpha, 23,24$-trimethyl- $5 \alpha$ cholest-22E-en-3 $\beta$-ol $\left./ n-\mathrm{C}_{20} \mathrm{OH}\right)_{\text {sediment }}$ 


\subsubsection{Stanol/stenol ratio}

Significant differences exist between the stanol/stenol ratios in suspended matter and sediments, involving lower stanol content in the former (Table 9). Similar differences between surface sediments and suspended particles have been observed elsewhere, e.g., the shelf off north Taiwan (Jeng and Huh, 2001). Suspended matter, which probably represents the lower stanol content of suspended particles, is consistent with their marine plankton origin (Volkman, 1986). The conversion of stenol to stanol by bacteria is expected to be slow in oxic surface waters. In contrast, the high stanol/stenol ratios of the shelf sediments may be caused by selective degradation of stenols relative to stanols at the

Table 8

Concentrations (ng/g) of alkanols and sterols for sediments

\begin{tabular}{|c|c|c|c|c|c|c|c|c|c|}
\hline \multirow[t]{2}{*}{ Compound } & \multicolumn{9}{|c|}{ Sample } \\
\hline & 10 & 14 & 18 & 20 & 22 & 24 & 32 & 34 & 36 \\
\hline \multicolumn{10}{|l|}{ Alkanols } \\
\hline$n-\mathrm{C}_{14} \mathrm{OH}$ & 5 & 11 & 10 & 14 & 17 & 24 & 14 & 19 & 19 \\
\hline$n-\mathrm{C}_{15} \mathrm{OH}$ & 3 & 9 & 6 & 9 & 13 & 15 & 9 & 14 & 12 \\
\hline$n-\mathrm{C}_{16} \mathrm{OH}$ & 20 & 56 & 42 & 58 & 75 & 97 & 70 & 84 & 67 \\
\hline$n-\mathrm{C}_{18} \mathrm{OH}$ & 10 & 55 & 39 & 59 & 63 & 48 & 53 & 60 & 68 \\
\hline phytol & 79 & 469 & 345 & 469 & 473 & 839 & 365 & 306 & 457 \\
\hline$n-\mathrm{C}_{19} \mathrm{OH}$ & 2 & 11 & 6 & 12 & 11 & 8 & 8 & 11 & 15 \\
\hline$n-\mathrm{C}_{20: 1} \mathrm{OH}$ & 2 & - & - & - & - & 41 & 21 & 15 & - \\
\hline$n-\mathrm{C}_{20} \mathrm{OH}$ & 9 & 83 & 42 & 89 & 74 & 40 & 68 & 83 & 82 \\
\hline$n-\mathrm{C}_{21} \mathrm{OH}$ & 2 & 22 & 10 & 24 & 18 & 12 & 19 & 22 & 25 \\
\hline$n-\mathrm{C}_{22: 1} \mathrm{OH}$ & 4 & 8 & 9 & 10 & 3 & 98 & 55 & 34 & 17 \\
\hline$n-\mathrm{C}_{22} \mathrm{OH}$ & 64 & 392 & 207 & 391 & 361 & 183 & 366 & 396 & 476 \\
\hline$n-\mathrm{C}_{23} \mathrm{OH}$ & 4 & 37 & 15 & 40 & 29 & 13 & 31 & 36 & 39 \\
\hline$n-\mathrm{C}_{24} \mathrm{OH}$ & 33 & 218 & 83 & 217 & 158 & 95 & 159 & 203 & 183 \\
\hline$n-\mathrm{C}_{25} \mathrm{OH}$ & 2 & 35 & 10 & 30 & 19 & 8 & 20 & 26 & 22 \\
\hline$n-\mathrm{C}_{26} \mathrm{OH}$ & 14 & 218 & 114 & 255 & 182 & 144 & 233 & 154 & 215 \\
\hline$n-\mathrm{C}_{27} \mathrm{OH}$ & 3 & 43 & 20 & 61 & 55 & 25 & 52 & 51 & 58 \\
\hline$n-\mathrm{C}_{28} \mathrm{OH}$ & 23 & 344 & 97 & 264 & 180 & 88 & 200 & 295 & 203 \\
\hline$n-\mathrm{C}_{29} \mathrm{OH}$ & - & - & - & - & - & - & - & - & - \\
\hline$n-\mathrm{C}_{30} \mathrm{OH}$ & - & - & 48 & 123 & 105 & - & 163 & 231 & 74 \\
\hline \multicolumn{10}{|l|}{ Sterols } \\
\hline A & - & - & 19 & 41 & 27 & 24 & 37 & 35 & 41 \\
\hline $\mathrm{B}$ & 23 & 85 & 63 & 83 & 55 & 152 & 197 & 78 & 101 \\
\hline $\mathrm{C}$ & - & - & 20 & 41 & 31 & 36 & 42 & 30 & 46 \\
\hline $\mathrm{D}$ & 43 & 349 & 203 & 245 & 185 & 238 & 365 & 195 & 348 \\
\hline $\mathrm{E}$ & 14 & 222 & 54 & 93 & 74 & 78 & 120 & 70 & 149 \\
\hline $\mathrm{F}$ & 165 & 1350 & 758 & 912 & 496 & 544 & 1300 & 727 & 1040 \\
\hline $\mathrm{G}$ & 34 & 382 & 179 & 358 & 229 & 145 & 241 & 228 & 333 \\
\hline $\mathrm{H}$ & 111 & 896 & 471 & 626 & 383 & 831 & 830 & 431 & 716 \\
\hline I & 29 & 309 & 118 & 225 & 155 & 147 & 153 & 153 & 217 \\
\hline $\mathbf{J}$ & 31 & 236 & 99 & 156 & 126 & 207 & 167 & 82 & 175 \\
\hline K & 41 & 360 & 152 & 230 & 189 & 224 & 230 & 179 & 227 \\
\hline $\mathrm{L}$ & 15 & 142 & 49 & 102 & 78 & 54 & 67 & 72 & 83 \\
\hline M & 22 & 272 & 71 & 108 & 105 & 129 & 101 & 98 & 139 \\
\hline $\mathrm{N}$ & 51 & 522 & 210 & 396 & 306 & 214 & 351 & 352 & 430 \\
\hline $\mathrm{O}$ & 26 & 250 & 86 & 189 & 146 & 162 & 187 & 149 & 207 \\
\hline $\mathrm{P}$ & 107 & 809 & 419 & 627 & 432 & 485 & 740 & 468 & 734 \\
\hline Q & 57 & 502 & 211 & 479 & 315 & 265 & 378 & 320 & 447 \\
\hline $\mathrm{R}$ & 60 & 730 & 311 & 605 & 523 & 502 & 881 & 939 & 567 \\
\hline
\end{tabular}

Sterol identifications: (A) 24-nor-5 $\alpha$-cholest-22E-en-3 $\beta$-ol, (B) 27-nor-24-methylcholesta-5,22E-dien-3 $\beta$-ol, (C) 27-nor-24-methyl-5 $\alpha$ -

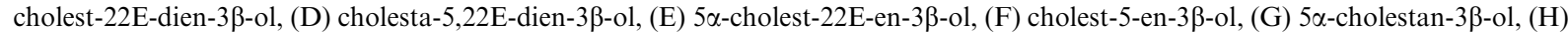

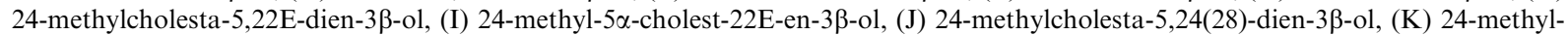

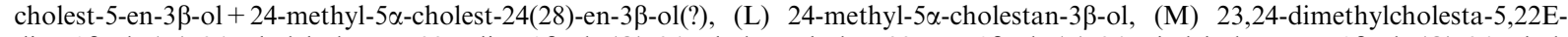

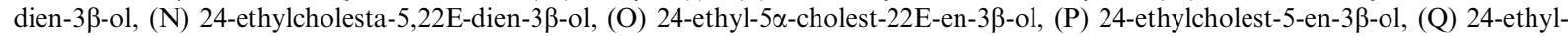

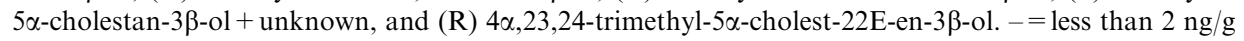




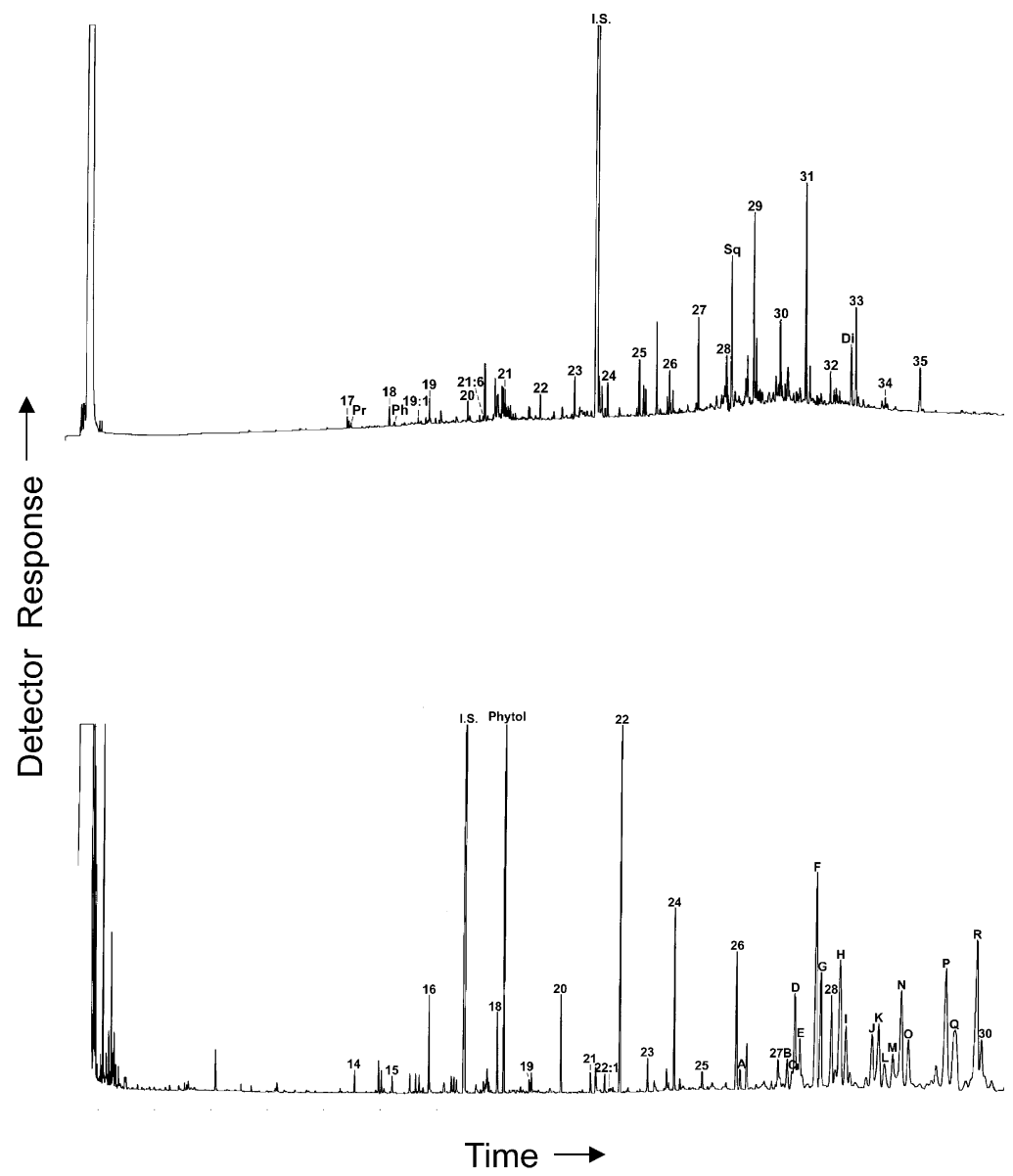

Fig. 3. (upper) Chromatogram of aliphatic hydrocarbons from the sediment of station 14. (lower) Chromatogram of the alkanol/ sterol fraction from the sediment of station 22. Sterol identifications as in Fig. 2.

Table 9

Stanol/stenol ratios for total suspended matter and sediments from the East China Sea. Note that numbers in parentheses are water depths

\begin{tabular}{|c|c|c|c|c|c|c|c|c|c|c|}
\hline Stanol/stenol & $\begin{array}{l}\text { Sta.10 } \\
(37 \mathrm{~m})\end{array}$ & $\begin{array}{l}\text { Sta.14 } \\
(60 \mathrm{~m})\end{array}$ & $\begin{array}{l}\text { Sta.18 } \\
(80 \mathrm{~m})\end{array}$ & $\begin{array}{l}\text { Sta.20 } \\
(65 \mathrm{~m})\end{array}$ & $\begin{array}{l}\text { Sta.22 } \\
(45 \mathrm{~m})\end{array}$ & $\begin{array}{l}\text { Sta.24 } \\
(32 \mathrm{~m})\end{array}$ & $\begin{array}{l}\text { Sta.32 } \\
(45 \mathrm{~m})\end{array}$ & $\begin{array}{l}\text { Sta.34 } \\
(40 \mathrm{~m})\end{array}$ & $\begin{array}{l}\text { Sta.36 } \\
(80 \mathrm{~m})\end{array}$ & Avg. \\
\hline \multicolumn{11}{|l|}{$T S M$} \\
\hline $\mathrm{E} / \mathrm{D}$ & 0.108 & 0.040 & 0.414 & 0.191 & 0.040 & 0.084 & 0.169 & 0.282 & 0.071 & 0.155 \\
\hline $\mathrm{G} / \mathrm{F}$ & 0.118 & 0.093 & 0.281 & 0.219 & 0.137 & 0.092 & 0.166 & 0.176 & 0.143 & 0.158 \\
\hline $\mathrm{I} / \mathrm{H}$ & 0.016 & 0.028 & 0.142 & 0.096 & 0.035 & 0.029 & 0.036 & 0.041 & 0.051 & 0.053 \\
\hline \multicolumn{11}{|l|}{ Sediment } \\
\hline $\mathrm{E} / \mathrm{D}$ & 0.326 & 0.636 & 0.266 & 0.380 & 0.400 & 0.328 & 0.329 & 0.359 & 0.428 & 0.384 \\
\hline $\mathrm{G} / \mathrm{F}$ & 0.206 & 0.283 & 0.236 & 0.393 & 0.462 & 0.267 & 0.185 & 0.314 & 0.320 & 0.296 \\
\hline $\mathrm{I} / \mathrm{H}$ & 0.261 & 0.345 & 0.251 & 0.359 & 0.415 & 0.177 & 0.184 & 0.355 & 0.303 & 0.294 \\
\hline
\end{tabular}

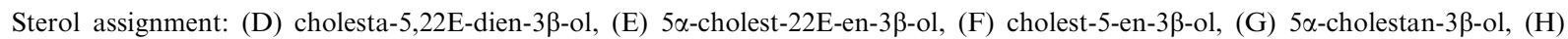

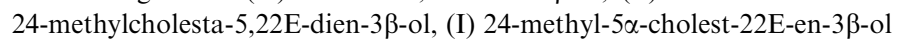


sediment/water interface (Nishimura and Koyama, 1977) or microbiological hydrogenation of stenols (Edmunds et al., 1980; Taylor et al., 1981).

\section{Conclusions}

The hydrocarbon, $n$-alkan-1-ol and sterol compositions of TSM from the ECS is dominated by marine planktonic inputs. High proportions of PDHCs appeared to be located at stations near the Changjiang River. Terrigenous lipids are not important contributors. The prevailing oxic conditions in the shelf water of the ECS contributed to the easy degradation of labile hydrocarbons and $n$-alkenols of planktonic origin.

The concentrations of lipids in TSM are correlated in different groups. The correlation of $n-\mathrm{C}_{17}, n-\mathrm{C}_{18}, n-\mathrm{C}_{19: 1}$ and $n-\mathrm{C}_{19}$ indicates a common source, namely phytoplankton species other than diatoms and copepods. Another group of correlated hydrocarbons is composed of $n-\mathrm{C}_{21: 6}$, pristane and squalene, suggesting a common source such as diatoms (microplankton). The close correlation between $\mathrm{C}_{16}, \mathrm{C}_{20: 1}$ and $\mathrm{C}_{22: 1}$ alcohols suggests a contribution from copepods. Most sterols are also correlated, being therefore attributed to marine sources.

Labile lipid biomarkers like PDHCs and monounsaturated alcohols dominated in TSM, but were only found in trace or minor amounts in the surface sediments. The prevailing oxic condition in the water column likely led to their degradation. Rough estimates of the relative degradation of these biomarkers in the ECS could be made using lipid data of TSM and of sediments. The sterol composition reflects predominant inputs from phytoplankton and zooplankton in TSM, and marine and terrigenous detritus, meiofauna and bivalves in sediments.

\section{Acknowledgements}

We are grateful to captain, crew and technicians of the R/V Ocean Researcher I for assistance with sample collection. Our special thanks go to two anonymous referees for constructive comments and suggestions, and to Professor J. O. Grimalt for editorial editing. This study was financially supported by a grant from the National Science Council, ROC (grant no. NSC90-2611-M-002-007).

\section{Associate Editor-J. Grimalt}

\section{References}

Albaiges, J., Grimalt, J., Bayona, J.M., Risebrough, R., De Lappe, B., Walker II., W., 1984. Dissolved, particulate and sedimentary hydrocarbons in a deltaic environment. Organic Geochemistry 6, 237-248.
Ballantine, J.A., Lavis, A., Morris, R.J., 1983. A seasonal survey of the sterol composition of the cockle Cerastoderma edule: a comparison of polluted and non-polluted environments. Marine Environmental Research 9, 111-122.

Barrett, S.M., Volkman, J.K., Dunstan, G.A., LeRoi, J.-M., 1995. Sterols of 14 species of marine diatoms (Bacillariophyta). Journal of Phycology 31, 360-369.

Beardsley, R.C., Limeburner, R., Yu, H., Cannon, G.A., 1985. Discharge of the Changjiang (Yangtze River) into the East China Sea. Continental Shelf Research 4, 57-76.

Blumer, M., Guillard, R.R.L., Chase, T., 1971. Hydrocarbons of marine phytoplankton. Marine Biology 8, 183-189.

Bouloubassi, I., Fillaux, J., Saliot, A., 2001. Hydrocarbons in surface sediments from the Changjiang (Yangtze River) Estuary, East China Sea. Marine Pollution Bulletin 42, 1335-1346.

Edmunds, K.L.H., Brassell, S.C., Eglinton, G., 1980. The short-term diagenetic fate of $5 \alpha$-cholestan- $3 \beta$-ol: in-situ radiolabelled incubations in algal mats. In: Douglas, A.G., Maxwell, J.R. (Eds.), Advances in Organic Geochemistry 1979. Pergamon Press, Oxford, pp. 427-434.

Gagosian, R.B., Peltzer, E.T., 1986. The importance of atmospheric input of terrestrial organic material to deep sea sediments. Organic Geochemistry 10, 661-669.

Gladu, P.K., Patterson, G.W., Wikfors, G.H., Chitwood, D.J., Lusby, W.R., 1991. Sterols of some diatoms. Phytochemistry 30, 2301-2303.

Goad, L.J., 1978. The sterols of marine invertebrates: composition, biosynthesis, and metabolites. In: Scheuer, P.J. (Ed.), Marine Natural Products: Chemical and Biological Perspectives, Vol. II. Academic Press, pp. 75-172.

Goad, L.J., Withers, N., 1982. Identification of 27-nor-(24R)24-methylcholesta-5,22-dien-3 $\beta$-ol and brassicasterol as the major sterols of the marine dinoflagellate Gymnodinium simplex. Lipids 17, 853-858.

Gong, G.C., Chen, Y.L., Liu, K.K., 1996. Chemical hydrography and chlorophyll $a$ distribution in the East China Sea in summer: implications in nutrient dynamics. Continental Shelf Research 16, 1561-1590.

Han, J., McCarthy, E.D., Van Hoeven, W., Calvin, M., Bradley, W.H., 1968. Organic geochemical studies, II. A preliminary report on the distribution of aliphatic hydrocarbons in algae, in bacteria, and in a recent lake sediment. Proceedings of the National Academy of Sciences (USA) 59, 29-33.

Harvey, H.R., 1994. Fatty acids and sterols as source markers of organic matter in sediments of the North Carolina continental slope. Deep-Sea Research II 41, 783-796.

Jeng, W.L., Huh, C.A., Chen, C.L., 1997. Alkanol and sterol degradation in a sediment core from the continental slope off southwestern Taiwan. Chemosphere 35, 2515-2523.

Jeng, W.L., Huh, C.A., 2001. Comparative study of sterols in shelf and slope sediments off northeastern Taiwan. Applied Geochemistry 16, 95-108.

Liu, H., He, D., 1990. A preliminary research on zooplankton in the Changjiang Estuary and its adjacent waters: biomass, composition, distribution and the heavy metals contents. In: $\mathrm{Yu}$ et al. (Eds.), Biogeochemical Study of the Changjiang Estuary. China Ocean Press, pp. 188-199.

Meyers, P.A., Leenheer, M.J., Eadie, B.J., Maule, S.J., 1984. Organic geochemistry of suspended and settling particulate matter in Lake Michigan. Geochimica et Cosmochimica Acta 48, 443-452. 
Milliman, J.D., Meade, R.H., 1983. World-wide delivery of river sediment to the oceans. Journal of Geology 91, 1-21.

Milliman, J.D., Shen, H.T., Yang, Z.S., Meade, R.H., 1985. Transport and deposition of river sediment in the Changjiang estuary and adjacent continental shelf. Continental Shelf Research 4, 37-45.

Milliman, J.D., Qin, Y.S., Park, Y.A., 1989. Sediments and sedimentary processes in the Yellow and East China Seas. In: Taira, A., Masuda, F. (Eds.), Sedimentary Facies in the Active Plate Margin. Terra Scientific Publishing Company, Tokyo, pp. 233-249.

Mudge, S.M., Norris, C.E., 1997. Lipid biomarkers in the Conwy (North Wales, UK): a comparison between fatty alcohols and sterols. Marine Chemistry 57, 61-84.

Nichols, P.D., Volkman, J.K., Palmisano, A.C., Smith, G.A., White, D.C., 1988. Occurrence of an isoprenoid $\mathrm{C}_{25}$ diunsaturated alkene and high neutral lipid content in Antarctic sea-ice diatom communities. Journal of Phycology 24, 9096.

Nishimura, M., Koyama, T., 1977. The occurrence of stanols in various living organisms and the behavior of sterols in contemporary sediments. Geochimica et Cosmochimica Acta 41, 379-385.

Oro, J., Tornabene, T.G., Nooner, D.W., Gelpi, E., 1967. Aliphatic hydrocarbons and fatty acids of some marine and freshwater microorganisms. Journal of Bacteriology 93, 1811-1818.

Osterroht, C., Petrick, G., 1982. Aliphatic hydrocarbons in particulate matter from the Baltic Sea. Marine Chemistry 11, 55-70.

Prahl, F.G., Bennett, J.T., Carpenter, R., 1980. The early diagenesis of aliphatic hydrocarbons and organic matter in sedimentary particulates from Dabob Bay, Washington. Geochimica et Cosmochimica Acta 44, 1967-1976.

Qiu, Y.J., Bigot, M., Saliot, A., 1991. Non-aromatic hydrocarbons in suspended matter from Changjiang (Yangtse River) Estuary: their characterization and variation in winter and summer (low- and high-flow) conditions. Estuarine, Coastal and Shelf Science 33, 153-174.

Quemeneur, M., Marty, Y., 1992. Sewage influence in a macrotidal estuary: fatty acid and sterol distributions. Estuarine, Coastal and Shelf Science 34, 347-363.

Saito, H., Kotani, Y., 2000. Lipids of four boreal species of calanoid copepods: origin of monoene fats of marine animals at higher trophic levels in the grazing food chain in the subarctic ocean ecosystem. Marine Chemistry 71, 69-82.

Saliot, A., 1981. Natural hydrocarbons in sea water. In: Duursma, E.K., Dawson, R. (Eds.), Marine Organic Chemistry. Elsevier, Amsterdam, pp. 327-374.

Saliot, A., Laureillard, J., Scribe, P., Sicre, M.A., 1991. Evolutionary trends in the lipid biomarker approach for investigating the biogeochemistry of organic matter in the marine environment. Marine Chemistry 36, 233-248.

Sargent, J.R., Lee, R.F., 1975. Biosynthesis of lipids in zooplankton from Saanich Inlet, British Columbia, Canada. Marine Biology 31, 15-23.

Schultz, D.M., Quinn, J.G., 1977. Suspended material in Narragansett Bay: fatty acid and hydrocarbon composition. Organic Geochemistry 1, 27-36.

Sicre, M.-A., Broyelle, I., Lorre, A., Saliot, A., 1993a. Sources and transport of particulate hydrocarbons in the meso-tidal Changjiang Estuary. Estuarine, Coastal and Shelf Science 37, 557-573.

Sicre, M.-A., Tian, R.C., Broyelle, I., Saliot, A., 1993 b. Aquatic distribution of 4-desmethyl sterols in the Chang Jiang Estuary, China. Marine Chemistry 42, 11-24.

Sicre, M.-A., Tian, R.C., Saliot, A., 1994. Distribution of sterols in the suspended particles of the Chang Jiang Estuary and adjacent East China Sea. Organic Geochemistry 21, 1-10.

Sun, M.Y., Wakeham, S.G., Aller, R.C., Lee, C., 1998. Impact of seasonal hypoxia on diagenesis of phytol and its derivatives in Long Island Sound. Marine Chemistry 62, 157-173.

Taylor, C.D., Smith, S.O., Gagosian, R.B., 1981. Use of microbial enrichments for the study of the anaerobic degradation of cholesterol. Geochimica et Cosmochimica Acta 45, 2161-2168.

Tian, R.C., Sicre, M.-A., Saliot, A., 1992. Aspects of the geochemistry of sedimentary sterols in the Chang Jiang Estuary. Organic Geochemistry 18, 843-850.

Tornabene, T.G., 1978. Non-aerated cultivation of Halobacterium cutirubrum and its effects on cellular squalenes. Journal of Molecular Evolution 11, 253-257.

Volkman, J.K., 1986. A review of sterol markers for marine and terrigenous organic matter. Organic Geochemistry 9, 83-99.

Volkman, J.K., Smith, D.J., Eglinton, G., Forsberg, T.E.V., Corner, E.D.S., 1981. Sterol and fatty acid composition of four marine Haptophycean algae. Journal of the Marine Biological Association of the U.K 61, 509-527.

Wakeham, S.G., 1995. Lipid biomarkers for heterotrophic alteration of suspended particulate organic matter in oxygenated and anoxic water columns of the ocean. Deep-Sea Research I 42, 1749-1771.

Wakeham, S.G., Lee, C., 1989. Organic geochemistry of particulate matter in the ocean: the role of particles in oceanic sedimentary cycles. Organic Geochemistry 14, 83-96.

Winters, K., Parker, P.L., Van Baalen, C., 1969. Hydrocarbons of blue-green algae: geochemical significance. Science 158, 467-468.

Wu, Y.L., Zhang, Y.S., Zhou, C.X., 2000. Phytoplankton distribution and community structure in the East China Sea (ECS) continental shelf. Chinese Journal of Oceanology and Limnology 18, 74-79. 